\title{
MASTER
}

WFPS: TME $-80-003$

JANUARY 1980

$U C-20$ A, D

\section{DETERMINATION OF NONAXISYMMETRIC EQUILIBRIUM}

\section{DAVID ELKIN}

\author{
COLUMBIA UNIVERSITY \\ APPLIED PHYSICS \& NUCLEAR ENGINEERING
}

$$
\text { aso } 9272
$$

COOPERATIVE GRADUATE EDUCATION PROGRAM IN FUSION TECHNOLOGY

ADMINISTERED FOR THE U.S. DEPARTMENT OF ENERGY

BY THE WESTINGHOUSE ELECTRIC CORPORATION

CONTRACT EG-77-C-02-4231-A000

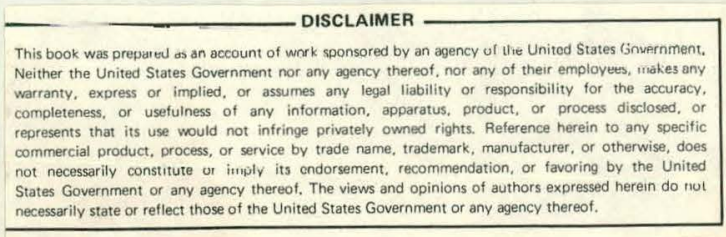




\section{DISCLAIMER}

This report was prepared as an account of work sponsored by an agency of the United States Government. Neither the United States Government nor any agency Thereof, nor any of their employees, makes any warranty, express or implied, or assumes any legal liability or responsibility for the accuracy, completeness, or usefulness of any information, apparatus, product, or process disclosed, or represents that its use would not infringe privately owned rights. Reference herein to any specific commercial product, process, or service by trade name, trademark, manufacturer, or otherwise does not necessarily constitute or imply its endorsement, recommendation, or favoring by the United States Government or any agency thereof. The views and opinions of authors expressed herein do not necessarily state or reflect those of the United States Government or any agency thereof. 


\section{DISCLAIMER}

Portions of this document may be illegible in electronic image products. Images are produced from the best available original document. 


\section{ACKNOWLEDGEMENT}

This work was performed for the U.S. Department of Energy, under Contract EG-77-C-02-4231-A000, Cooperative Graduate Education Program in Fusion Technology. Reproduction, translation, publication, use and disposal, in whole or in part, by or for the United States Government is permitted.

This work depends strongly upon the previous attainments of others. If contributions towards the same goal of deriving the benefits of fusion are able to stem from this branch my gratitude to my advisors shall therein be best expressed. They, to whom I owe deep appreciation, are Dr. J. L. Johnson, for suggesting the problem and providing ready assistance and guidance, and Dr. T. F. Yang, for his direct supervision and encouragement though his major responsibilities were in another field. For conveying a portion of their insight into physics and demonstrating their manner of conducting research I am indebted. My further appreciation is extended to Dr. R. A. Gross for his assistance as I crossed over the threshold into special research.

I was given assistance during my stay at the Westinghouse Electric Corp. by many of the engineers and scientists. I thank Mrs. E. Iwinski for her invaluable aid in treating problems in programming, and I thank Dr. G. Gibson and Dr. D. Klein for their courtesy and suggestions.

Lastly, I am grateful for the support provided on the graduate level by the Department of Energy.

\section{NOT ICE}

This report was prepared as an account of work sponsored by an agency of the United States Government. Neither the United States nor any agency thereof, nor any of their employees, makes any warranty, expressed or implied, or assumes any legal liability or responsibility for any third party's use or the results of such use of any information, apparatus, product or process disclosed in this report, or represents that its use by such third party would not infringe privately owned rights.

Printed in the United States of America Available From

National Technical Information Service

U.S. Department of Commerce

5285 Port Royal Road

Springfield VA 22161

\section{NTIS Price Codes}

Printed Copy: A03

Microfiche Copy: A01 
WFPS:TME-80-003

JANUARY 1980

$U C-20$ a,g

\section{DETERMINATION OF NONAXISYMMETRIC EQUILIBRIUM}
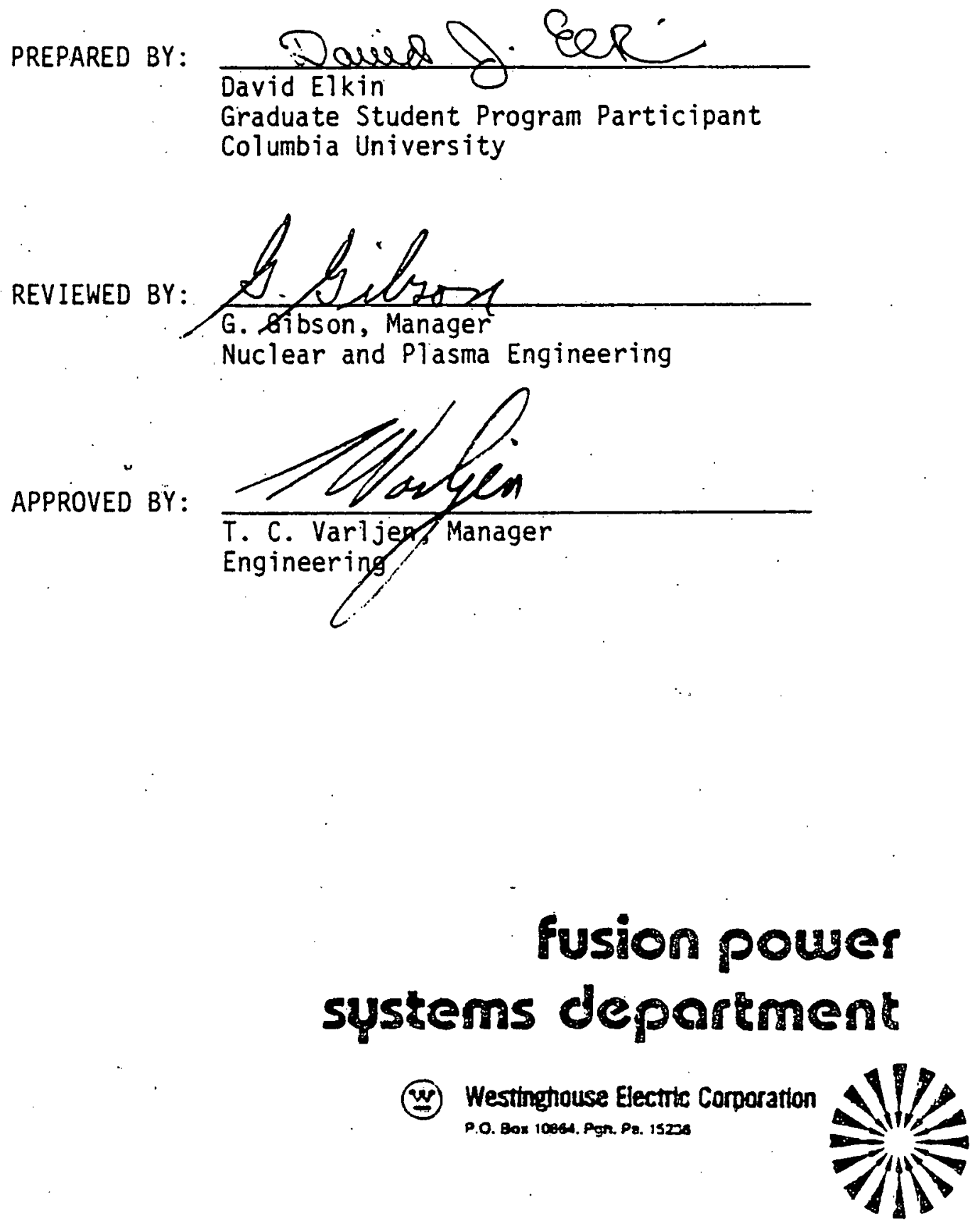
To Miss Sherry M. Meltzer

for her faithful concern. 
Wise in his daily work was he:

To fruits of diligence,

And not to faiths or polity,

He plied his utmost sense.

These perfect in their little parts,

Whose work is all their prize --

Without them how could laws, or arts,

or towered cities, rise?

Middlemarch, Ch. 40 
Abstract

The Princeton Equilibrium Code is modified to determine the equilibrium surfaces for a large aspect ratio toroidal system with helical magnetic fields. The code may easily be made to include any variety of modes. Verification of the code is made by comparison with an analytic solution for $\ell=3$. Previously observed shifting of the magnetic axis. with increasing pressure or with a changed externally applied vertical field is obtained. The case $\ell=0$, a bumpy torus, gives convergence only for the lenient convergence tolerance of $\varepsilon_{b}=1.0 \times 10^{-2}$. 


\section{TABLE OF CONTENTS}

Page

$\begin{aligned} \text { I } & \text { INTRODUCTION } \\ \text { II } & \text { IMPLEMENTATION } \\ \text { II } & \text { CODE VALIDATION } \\ \text { IV } & \text { ADDITIONAL APPLICATIONS AND DISCUSSION } \\ V & \text { APPENDIX } \\ & \text { REFERENCES }\end{aligned}$

1

5

7

9

11

16 


\section{LIST OF TABLES}

Table No.

Page

BASIC EQUATIONS

17

\section{LIST OF FIGURES}

Figure No.

Page

Coordinates of the Torus of Major Radius R, Minor Radius a

2 Coordinates for the Grid

3a. Comparison of Analytical and Computational Solutions for $B=0 \quad 25$

3b Comparison of Analytical and Computational Solutions for $B \bar{\tau} .1 \%$

4a Contours of Poloidal Magnetic Flux: $\beta=0 \quad 27$

$4 b \quad$ Contours of Plasma Current Along Field Lines: $\beta=0 \quad 28$

4c Current Profile at the Grid Midplane: $\beta=0 \quad 29$

$4 d \quad$ Linear Pressure Profile $\quad 30$

$4 \mathrm{e}$ : Contours of Poloidal Magnetic Flux: $\beta=2 \% \quad 31$

$4 f$ Contours of Plasma Current Along Field Lines: $\beta=2 \% \quad 32$

$4 \mathrm{~g}$ Current Profile at the Grid Midplane: $\beta=2 \%$

4h Pressure Profile at the Grid Midplane: $\beta=2 \% \quad 34$

5 Position of Magnetic Axis vs $P_{\max } \quad 35$

6 Position of Magnetic Axis vs Current in Vertical Field Coils 36 


\section{Introduction}

The departure from perfect symmetry in an operating toroidal plasma confinement device is a recognized necessity in the placement of toroidal field coils; to permit the functions of external heating and diagnostics, and to establish particular characteristics of the magnetic field useful for confinement and control of instabilities. In tokamaks the nonaxisymmetric effect of ripple fields is of interest. In bumpy tori a departure from periodicity in addition to the nonaxisymmetric features could intensify the problem of global field errors. A model for a confined plasma in a helically invariant magnetic field has been developed. ${ }^{1}$. We have incorporated it into an axisymmetric toroidal equilibrium code. ${ }^{2}$ The problem of short wavelength perturbations from toroidal symmetry is one immediate application for the modified code.

A confined plasma in equilibrium in a toroidal system is established by a magnetic field pattern which produces magnetic surfaces. The magnetic lines of force lie upon the magnetic surfaces and cover them densely. In so doing they provide the means for confinement. Orift across magnetic surfaces is caused by the usual toroidal forces -- a pressure gradient resulting in a net outward force, the Lorentz. force upon poloidal current, and charge separation due to the $1 / r$ dependence of the field. Curvature and inhomogeneilies in the magnetic field produce drift, and diffusion resulting from particle collisions contributes as well to the loss of confined plasma. If the time scale for drift across a magnetic surface is small compared with the time interval in which a particle may follow a field line and sample an entire magnetic surface then the average drift is made to vanish by the symmetry of the field lines. The local drifts are balanced -- particles which tend to leave the confinement region at the top re-enter at the bottom. In a 
high temperature plasma the time scale for diffusion would be long and the plasma would be expected to diffuse through a series of static equilibria. The existence of magnetic surfaces even for the vacuum system is assured by the assumption of periodicity. A line of force remains close to a magnetic surface to a degree given by an arbitrary power of the rotational transform. ${ }^{3}$ If, over a period, the rotational transform is small, the existence of magnetic surfaces, which are also surfaces of constant pressure, can be proved and the surfaces determined to arbitrary accuracy.

The equation of motion reduces to

$$
\vec{\nabla} P=\vec{J} \times \vec{B}
$$

for static equilibria. The plasma is approximated as a single fluid and the dispersion of individual particles is neglected. A scalar pressure is assumed. We use Maxwell's Equations

$$
\vec{\nabla} \cdot \vec{B}=0 \quad \vec{\nabla} \times \vec{B}=4 \pi \vec{J}
$$

and neglect the displacement current. Kruskal and Kulsrud ${ }^{4}$ discuss a variational principle to solve the equilibrium equations. In their work the existence of a set of nested toroidal magnetic surfaces is a constraint of the variation. Here, a differential formalism results from the assumption of magnetic surfaces labelled by a coördinate $\psi$ :

$$
\vec{B} \cdot \vec{\nabla} \psi=0
$$

A magnetic surface is also a constant current surface -- there is no current perpendicular to the surface in the limit of low amounts of diffusion. The physical mechanism of equilibrium that obtains depends upon the current in the plasma to support the pressure (current perpendicular to the magnetic field) and to redistribute the accumulated charge quenching the causes of 
drift (parallel current).

A zeroth order uniform longitudinal field is assumed in the stellarator model? with the fields associated with curvature, current in the external helical windings, and plasma current small in comparison with the axial field. A steady-state system is envisioned wherein current along a magnetic field line may exist microscopically but net current through a cross-section of the torus vanishes.

In the left-handed system of Fig. 1

$$
d l^{2}=d r^{2}+r^{2} d \theta^{2}+(1-k r \cos \theta)^{2} d \zeta^{2}
$$

Define $\delta$ and $\beta$ as quantities that represent the strength of the helical field and the amount of plasma according to

$$
\begin{aligned}
& r=a(1+\delta \cos (l \theta-h \zeta)) . \\
& \beta=8 \pi P(0) \mid B^{(0)^{2}}
\end{aligned}
$$

so that all quantities may be expanded in powers of these parameters. The appropriate ordering sets $\beta, \kappa, \sigma, \delta \delta$ as second order quantities. These represent the fields associated with plasma pressure, curvature, parallel current, and higher order terms in the multi-polar windings.

$$
\left.\left.\left(B^{(\delta)} \mid \cdot B^{(0)}\right)^{2} \sim B^{(P)}\right|_{B^{(0)}} \sim B^{(k)}\left|B^{(0)} \sim B^{(\sigma)}\right| B^{(0)} \sim B^{(\delta \delta)}\right|_{B^{(0)}} \sim \epsilon \equiv / R<1 .
$$

A derivation of the expression for $\psi^{(0)}$ where a single helical field mode is present is given in the appendix, along with the helically invariant refinement given by $\psi^{(1)}(r, \theta, \zeta)$. The equations of the model are summarized in Table 1.

In second order, the lowest significant order, a two-dimensional equation for $A^{(\sigma)}$ is obtained, being a nonlinear elliptic partial differential equation. 
The tokamak equilibrium code, ${ }^{2}$ which was designed to solve the Grad-Shafranov equation, can thus be modified for application to this problem. A comparison with the tokamak equilibrium problem, as shown in Table 2, reveals the following :

1) An algebraic relation exists between the surface coördinate and the appropriate coördinate of the vector potential in both cases. The surface coördinate is equivalent to the poloidal flux function. The effect of the helical windings is to add the term with modified Bessel functions, which is an increasing function of radius.

2) The differential operator in the multipolar case reduces to a Laplacian operator because of the large aspect ratio expansion.

3) $\sigma B^{(0)}$ on the right hand side represents current parallel to a field line.

4). The current in both cases is a sum of two terms:

- pressure gradient with a geometrical factor associated with field line curvature

- an arbitrary net current profile.

5.) Note the absence of the $\zeta$ dependence in the differential equation.

Let us examine the algorithm for the Princeton Equilibrium Code $^{2}$ as a prelude for discussion of its modification. A finite grid $(33 \times 33,65 \times 65$, $124 \times 124)$ is chosen and an initialization of the poloidal flux values is made. From this a determination of the initial current distribution is irmediate. Dirichlet boundary conditions are then computed using a Green's function for a toroidal current source.: The contribution from external coils is included here. An iterative procedure is then followed to solve the Grad-Shafranov 
equation, which improves the values of the flux and current until convergence is obtained. The simple profiles

L - Limiter

M - Magnetic Axis

$$
\begin{aligned}
& p=p_{0}\left(\frac{\psi_{L}-\psi}{\psi_{L}-\psi_{m}}\right)^{\alpha} \\
& g=1-\gamma\left(\frac{\psi_{L}-\psi}{\psi_{L}-\psi_{m}}\right) \gamma^{\beta}
\end{aligned}
$$

are usually used. Centered difference equations approximate the differential equation on the mesh and double cyclic reduction ${ }^{5}$ is used. The plasma vacuum interface is determined by material or magnetic limiters. An inner loop calculates values of flux and current with fixed boundary conditions while an outer loop updates boundary values. Convergence of the boundary values is defined when an input tolerance, $\varepsilon_{b}$, is given and

$$
\left|\frac{\psi^{m}-\psi^{m-1}}{\psi_{L}-\psi_{m}}\right| \leqslant 5 \epsilon_{b}
$$

for each boundary point and the average value, BERR, is less than $\varepsilon_{b}$. The inner loop convergence criterion is $\frac{1}{10}$ BERR or $\varepsilon_{b}$, whichever is greater. An input tolerance of $10^{-4}$ or better is desirable to establish convergence. Upon obtaining a solution the following plots are constructed: pressure vs. $\psi, g$ function and $q$ function vs. $\Psi$, contours of poloidal flux, contours of toroidal plasma current, midplane current profile, midplane pressure profile.

\section{Implementation}

The modifications necessary to incorporate the helical field system ${ }^{1}$ into the Princeton Equilibrium Code $^{2}$ then are: 
1) Calculation of the vector potential and then addition of an algebraic function to determine the surface function $\psi(0)$.

2) Incorporation of curvature terms $\Omega$ into the calculation of $\sigma$, the parallel current.

3) Modification of the differential operator fram $\nabla^{*}$ to $\nabla^{2}$, together with modification of the associated Green's function.

For the purpose of this implementation the dependences of $p$ and $G$ on ${ }_{\psi}(0)$ were assumed to be linear;

$$
\begin{aligned}
& p=p_{0}\left(1-\alpha \psi^{(0)}\right) \\
& G=G 0+k G 1 \psi^{(0)} / 8 \pi^{2} B^{(0)}
\end{aligned}
$$

with GO determined to make the net current along the field in the plasma vanish. Other dependence is possible and will have to be incorporated to make the net current vanish on more than one surface.

The initialization chosen for the calculation of a single-mode system is just the flux function with $A$ set equal to zero,

$$
\psi_{i m i t i a)}^{(0)}(r, 8)=\frac{\pi B^{(0)}}{k} \frac{\epsilon_{m}^{2}}{h^{2} r} I_{m}^{\prime}(h r) I_{m}(h r) \text {. }
$$

In place of the convergence test

we used

$$
\left|\frac{\psi^{m}-\psi^{m-1}}{\psi_{L}-\psi_{m}}\right| \leq 5 \varepsilon_{b}
$$

$$
\left|\frac{A^{m}-A^{m-1}}{\psi_{L}-\psi_{m}}\right| \leqslant 5 \leqslant b
$$

with $\varepsilon_{b}=10^{-16}$ to $10^{-20}$. This is an equivalent form that avoids the round-off errors likely to occur for small $A$.

Values for the following parameters and quantities are required as input to the code: 
7

$B^{0}$

epsconv

$p^{0}$

$\operatorname{expp}$

gil

nim

rzero

a lr

alt

uncoils...

hims...

nulls... longitudinal magnetic field

$s_{b}$

pressure maximum

exponent of pressure profile

free parameter in current profile

$33,65,124$

distance to inside boundary of grid

horizontal grid dimension

vertical grid dimension

external coil information

number and position of material 1 imiters

number and position of magnetic limiters.

We worked in a system with $r=20, a] r=a] z=2, B^{\circ}=1, g g l=-200$, nd im=33, null $s=0$,

$n$ lims $=2$ (each 0.1 from the edge on the midplane with a height of 0.1 ).

III. Code Validation

Verification that the code is working correctly was obtained by treating a case with a pure $\ell=3$. field and with $p$ and $G$ linear functions of ${ }_{\Psi}(0)$. For this case a quadrature solution is available, ${ }^{1}$ so that an exact comparison of the computational results with analysis can be made. Thus,

$$
\begin{aligned}
& \Psi^{(0)}=\frac{\pi B^{(0)}}{k}\left(\frac{3 \epsilon^{2}}{h^{2} r} I_{3}^{\prime}(h r) I_{3}(h r)\right)
\end{aligned}
$$

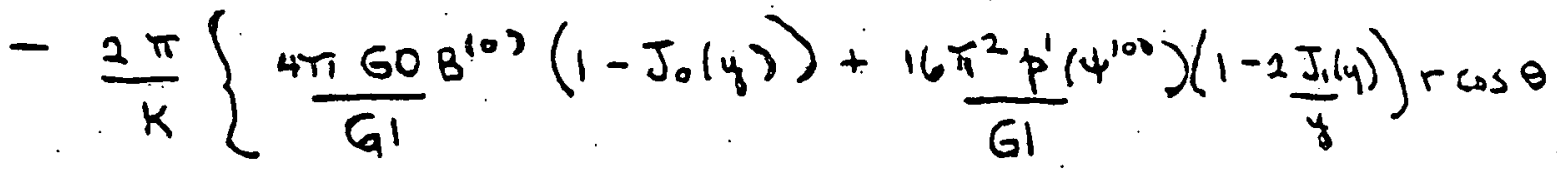

$$
\begin{aligned}
& \begin{array}{l}
-\frac{\pi}{4}\left(Y_{0}(y) \int_{0}^{r} J_{0}\left(y^{\prime}\right) \Gamma\left(r^{\prime}\right) r^{\prime} d r^{\prime}\right. \\
\left.\left.\quad-J_{0}(y) \int_{0}^{r} Y_{0}\left(y^{\prime}\right) \Gamma\left(r^{\prime}\right) r^{\prime} d r^{\prime}\right)\right\}
\end{array} \\
& \Gamma(r)=\epsilon^{2} I_{3}^{\prime}(h r)\left\{-\frac{8 \pi^{2} \psi^{\prime}}{k}\left(\psi^{(0)}\right)\left(1+\left(q+h^{2} r^{2}\right) I^{3}(h r)\right)\right. \\
& +3\left(6 / 8^{(0)} I^{3}(h r) \mid h\right\}
\end{aligned}
$$


$G 1<0$

$$
y=|G 1|^{1 / 2}+
$$

The quadratures were computed using the extended Simpson's rule

$$
\begin{array}{r}
\int_{x_{0}}^{x_{2 n}} f=\frac{h}{3}\left[f_{0}+4\left(f_{1}+f_{3}+\ldots\right)\right. \\
\quad+2\left(f_{2}+f_{4}+\cdots\right) \\
h=x_{i+1}-x_{i}
\end{array}
$$

Increasing $n$ had no effect on the result for $n>18$. Polynomial approximations were used for the computation of the Bessel functions.

Results for $p Q=0$ and.. $p 0=3$ are given in Table 3 , where a comparison is made atong the vertical midplane. The meaning of the indices $i, j$ is given in the accompanying chart - Fig. 2. The constant of integration for $\psi^{(0)}$ has been chosen so that the values at $(17,17)$, the center of the grid, agree. Values of the vector potential are shown. In the comparison at $p=0$ the $a b-$ solute difference at $j=5$ is $0.019 \times 10^{-11}$, or $0.13 \%$ using the value at $j=17$ as base. Disagreement for lower values of $j$ is slight. This data is presented in Figures $3 a$ and $3 b$ where the difference between the solutions obtained is noticeable only in the outer region at the limiter.

Confirmation of the shift of magnetic axis caused by pressure and vertical magnetic fields, observed in Ref. 1 , is demonstrated in Figures $4 a, 4 e, 5$, 6. A vacuum system gives rise to circular, concentric poloidal flux contours because of the symmetry which is present in two dimensions. With increasing pressure the magnetic axis shifts outwards while the contour at the boundary is held nearly fixed. At the edge of the domain the major contribution to the flux function, is from the helical windings while near the helical axis 
where the helical fields are small the vector potential becomes important. We recall the earlier discussion concerning toroidal forces and note here that since the contribution to the current is of the form

$$
-p^{\prime}\left(\psi^{(0)}\right) \Omega(r, \theta)
$$

where $\Omega$ incorporates curvature, positive current and-hence a positive contribution by $A$ obtains in most of the outward lying grid points. The magnetic axis shifts in an outward direction as expected by our previous discussion. The pressure of the fluid is balanced by the concentration of magnetic field energy density as is evidenced by the bunching of contour surfaces. A limit on the possible $\beta$ is given by the most extreme equilibrium state possible. The outward shift can be changed by introduction of an external vertical field. The vertical magnetic field superimposes upon the poloidal magnetic field, rainforcing or diminishing it according to its direction. An upwards pointing field of proper magnitude wi11, as shown in Ref. 1, nearly eliminate the secondary currents. In the presence of a vertical field at an intensity of $5 \%$ of $B^{(0)}$, a change of $B$ from zero to $2.5 \%$ shows negligible effect on the surface shape. Figures $4 a$ to 4 h show the contours of poloidal flux and current, pressure and current density profiles for $\beta=0$ and $\beta=2 \%$. Figure 5 shows the shift in location of the magnetic axis with pressure. Figure 6 shows the effect of the vertical field and that it is independent of pressure.

\section{Additional Appilications and Discussion}

A possible application of the program would be to the study of a bumpy torus with oniy an $\ell=0$ mode present. Here

$$
\begin{aligned}
\psi^{(0)}= & -\frac{2 \pi}{k} A^{(r)} \\
\Omega= & \frac{1}{2} \epsilon_{0}^{2}\left(\left(I_{0}^{\prime}(h r)\right)^{2}+\left(I_{0}(h r)\right)^{2}\right) \\
& -2 k r \cos \theta .
\end{aligned}
$$


In this case, since there is no rotational transform present, the $\psi^{(0)}$ surfaces, which are surfaces of constant pressure, must be functions of ${ }^{1,6}$

$$
u=\oint d l \mid B=\frac{2 \pi}{h B^{(0)}}\left(1+\Omega+4 \pi p / B^{(0)^{2}}\right) \text {. }
$$

That solutions should be found has been demonstrated recently. ${ }^{7}$

The difficulty in obtaining such equilibria is in making the toroidal current vanish on each $\S d \ell / B$ surface. We have only obtained vanishing net toroidal current since we have restricted $G$ to a linear function of $\psi(0)$, and have allowed the existence of local currents. These have dominated the solution for $\psi^{(0)}$ with convergence obtained for $\varepsilon_{b}=10^{-2}$. A polynomial form for $G$ may provide enough adjustable parameters so that the net current on each magnetic surface can be brought close to zero and a smaller value of $\varepsilon_{b}$ yield convergence.

Clearly a more sophisticated method for choosing $G\left(\psi^{(0)}\right)$ must be developed if this application is to be made. If such a technique were found, it would be interesting to see how large an $\ell=1$ error field, which resonates with the bumpy fields, could be introduced before it drastically changed the configuration. The code is capable of receiving any combination of helical modes as indicated in the equations for $\psi^{\circ}$ and $\Omega$ in Table 1 . Equilibria for a variety of systems with helical fields may be contemplated and the effects of small nelical perturbations studied.

A more immediate application that could and will be pursued is to apply the code to the study of equilibrium properties of the modular twisted torus configurations which have recentily received new interest. ${ }^{8}$ Continuation of this type of work could take several directions. As has been noted, ${ }^{9}$ it would be straightforward to implement a program to study ballooning modes in stellarator configurations similar to that used for tokamaks, ${ }^{10}$ or even to treat lower mode number instabilities as in the PEST program. ${ }^{11}$ A different equilibrium formalism, using a guiding-center formulation ${ }^{12}$ so as to incorporate a better treatment of the effect of nonisotropic pressure or of particle trapping in the helical fields, would be useful. 
Appendix

11

I) Determinition of $\psi^{(0)}$ for a pire nole $l$.

$$
\begin{aligned}
& \vec{B}=\vec{B}^{(0)}+\vec{B}^{(1)}+\vec{B}^{(2)} \\
& \vec{B}=B^{(0)} \hat{z}+\vec{\nabla} \frac{\epsilon_{1} B^{(0)}}{h} I_{2}\left(h_{r}\right) \sin w \\
& +\left(B^{(P)}+B^{(k)}\right) \hat{z}-\hat{E} \times \vec{\nabla} A+B^{(\delta \Sigma)} \\
& \vec{B}^{(\sigma)}(r, \theta) \perp \hat{z} \quad \vec{B}^{(\sigma)}=\nabla \times A \hat{z}=-\hat{z} \times J A
\end{aligned}
$$

$u=C \theta-h z \quad \vec{A}=(0,0, A)$ supessenpt $(\sigma)$ is suppressed.

$$
\begin{aligned}
& \vec{B} \cdot \vec{\nabla} \psi^{(0)}=0, \sum_{n=0}^{m} B^{(n)} \cdot \nabla \Psi^{(m-n)}=0 \\
& n_{1}=0 \quad B^{(0)} \partial_{z} \psi^{(0)}=0 \Rightarrow \psi^{(0)}=\psi^{(0)}(r, 0) \\
& n=1 \quad B^{(0)} \partial_{z} \psi^{(1)}+\frac{E_{2} B^{(i)}}{h} \cdot \vec{\nabla} I_{1} \sin u \cdot \vec{\nabla} \psi^{(0)}=0 \\
& \psi^{(1)}=-\frac{\epsilon_{2}}{h^{2}} \nabla I_{l} \cos u \cdot \vec{\nabla} \psi^{(0)}+\Gamma^{(1)}(r, \theta) \\
& m=2 \quad B^{(0)} \partial z \psi^{(r)}+\frac{\epsilon_{z} B^{(0)}}{h} \nabla I_{1} \sin u \\
& \cdot \vec{\nabla}\left[-\frac{\epsilon_{0}}{h^{2}} \vec{\nabla} I_{l} \cos u \cdot \sigma \psi(0)+\Gamma^{(1)}(r, \theta)\right] \\
& +\left(\overrightarrow{B^{\prime}}(\beta)+\vec{B}^{(k)}+\vec{B}(\delta \Sigma)\right) \cdot \vec{\nabla} \psi^{(0)} \\
& -\hat{z} \times \vec{\nabla} A \cdot \vec{\nabla} \psi^{(0)}=0
\end{aligned}
$$

Shi pelicilienty condition demenels tiut

$$
\begin{aligned}
& <-\frac{E^{2} B^{(0)}}{h^{3}} \vec{\nabla} I_{l} \sin n \cdot \vec{\nabla}\left[\left(\vec{\nabla} I_{l}(\cos n) \cdot \vec{\nabla} \psi^{(0)}\right]\right. \\
& \left.-\vec{z} \times \vec{\nabla} A \cdot \vec{\nabla} \psi^{(\omega)}\right\rangle=0
\end{aligned}
$$


12

a period.

$$
\begin{aligned}
& \left\langle-\frac{\epsilon_{0}^{2} B^{(0)}}{h^{3}}\left\{\hat{r} h I_{l}^{\prime} \sin u+\frac{\hat{\theta} l I_{l}}{r} \cos u-\hat{z} h I_{Q} \cos u\right\}\right. \\
& \text { - } \vec{\nabla}\left\{\left(\hat{r} h I_{q}^{\prime} \cos u-\frac{\hat{\theta} l}{r} I_{l} \sin u\right) \cdot \vec{\nabla} \psi^{(0)}\right\} \\
& \left.-\hat{z} \times \vec{\nabla} A \cdot \vec{\nabla} \psi^{(0)} .\right\rangle=0^{1}
\end{aligned}
$$

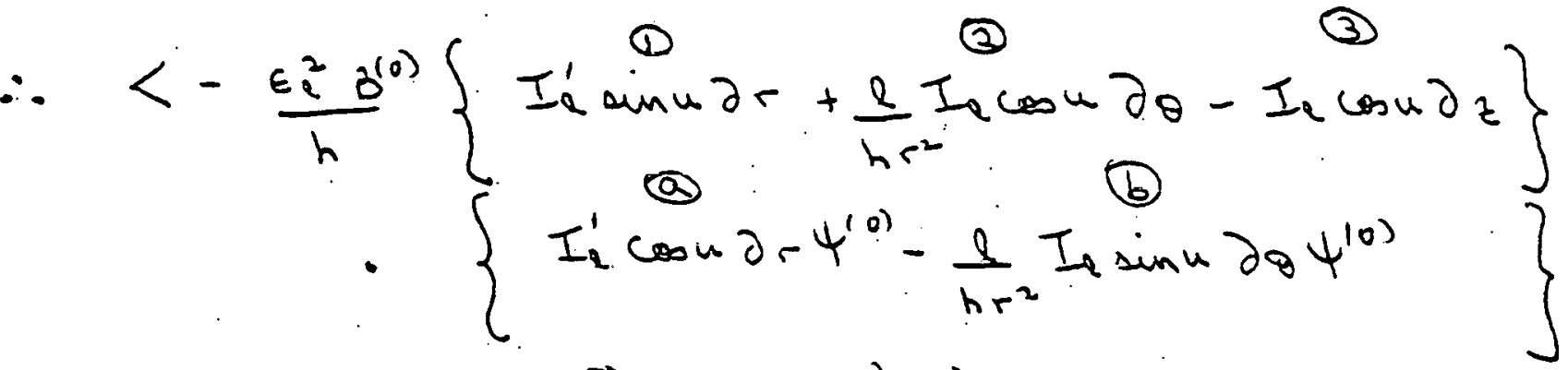

$$
\begin{aligned}
& \left.-\hat{z} \times \vec{\nabla} A \cdot \nabla \psi^{(0)}\right\rangle=0
\end{aligned}
$$

Shoes: I! $\equiv \frac{d}{d} \frac{I_{l}\left(h_{r}\right)}{\left(h_{r}\right)}$.

la $I_{l}^{\prime} \partial_{r}\left(I^{\prime} \partial_{r} \psi^{(0)}\right) \cos u \sin w$

ib: $\left\{-I_{l}^{\prime} \frac{e}{h} \partial r\left(\frac{I_{Q}}{r^{2}} \partial \theta \psi^{(0)}\right) \sin ^{2} u\right\}$

aa. $\frac{l}{h r^{2}} I_{R} I_{2}^{\prime} \cdot \partial_{\theta}\left(\cos \omega \partial_{r} \psi^{(0)}\right) \cos u$

$2 b \quad-\frac{l^{2}}{h^{3} r^{4}} I^{2} \partial \theta\left(\sin u \partial \theta \psi^{(0)}\right) \cos \omega$

sa- Ie I $I^{\prime} h \partial r \psi^{(0)} \sin n \cos k$

$$
\begin{aligned}
& \text { sb } \quad-I_{l}^{2} \frac{e \cos ^{2} u \partial \theta \psi^{(0)}}{r^{2}} \\
& -\frac{1}{r} \partial r A \partial \theta \psi^{(0)}+\frac{1}{r} \partial \theta A \partial=\psi^{(0)}
\end{aligned}
$$


13

Queroging over a perial, $\overline{\sin ^{2}}=\overline{\cos ^{2}}=1 / 2$ and sinces $=0$, we optain

$$
\begin{aligned}
& -\frac{\epsilon_{l}^{2} B^{(0)}}{2 h}\left(-I_{l}^{1} \frac{\partial}{\lambda} \partial r\left(\frac{I_{l}}{r^{2}} \partial \theta \psi^{(0)}\right)+\frac{Q}{h r^{2}} I_{l} I_{l}^{\prime} \partial r \theta \psi^{(0)}\right. \\
& \left.-\frac{l^{3}}{h^{2} r^{4}} I_{l}^{2} \partial \theta \psi^{(0)}-\frac{e I_{l}^{2}}{r^{2}} \partial \theta \psi^{(0)}\right) \\
& -\frac{1}{r} \partial r A \partial \theta \psi^{(0)}+\frac{1}{r} \partial \theta A \partial r \psi^{(0)}=0 \text {. }
\end{aligned}
$$

On pist andien

$$
\begin{aligned}
& \left\{\frac{l E_{i}^{2} B^{(0)}}{2 h r}\left(\frac{h I_{0}^{\prime 2}}{h}-\frac{2 I_{l}^{\prime} I_{l}}{h r}+\left(1+\frac{l^{2}}{h^{2} r^{2}}\right) \frac{I_{l}^{2}}{r}\right)-\partial r A\right\} \perp \partial_{r} \psi^{(0)} \\
& +\frac{1}{r} \partial_{\theta} A \partial r \psi^{(0)}=0 \text {. } \\
& \therefore \vec{F} \cdot \vec{\nabla} \psi^{(0)}=0 \\
& F_{r}=\frac{2 \pi}{k} \perp_{r} \partial_{\theta} A \\
& F_{\theta}=\frac{\pi B^{(0)}}{k} \frac{\epsilon_{l}^{2} l}{h r}\left(I_{l}^{2}-\frac{2 I_{l}^{1} I_{l}}{h r}+\left(1+\frac{l^{2}}{h^{2} r^{2}}\right) I_{l}^{2}\right)-\frac{2 \pi}{k} \partial_{r} A \\
& -F_{r} r=\partial_{\theta} \psi^{(0)}=-\frac{2 \pi}{k} \partial \theta A, \quad \partial_{r} \psi^{(0)}=F_{\theta} \\
& \therefore \psi^{(0)}=\frac{\pi B^{(0)}}{k} \frac{E_{l}^{2} l}{h^{2} r} I_{Q}^{\prime} I_{l}-\frac{2 \pi}{k} A \text {. }
\end{aligned}
$$
The derintion for $\sigma^{(\lambda \lambda)}$ fallows an avelogous
sente. 
14

II) Determintion of fubial ufinement to $\psi^{(0)}$ by $\psi^{(1)}$.

$$
\begin{aligned}
& B^{(0)} \partial z \psi^{(1)}=-\vec{B}^{(1)} \cdot \vec{\nabla} \psi^{(0)} \\
& \psi^{(1)}=-\frac{1}{B^{(0)}} \int \vec{B}^{(1)} d z \cdot \vec{\nabla} \psi^{(0)}+\Gamma^{(1)}(r, \theta)
\end{aligned}
$$

We ignore $\Gamma^{(1)}$ as not praviding a fulieally invorient contivitution :

$$
\begin{aligned}
\vec{B}^{(1)}= & \frac{\epsilon_{Q} B^{(0)}}{h} \vec{\nabla} I_{l} \sin u \\
& \int \vec{B}_{\perp}^{(1)} d z=\frac{\epsilon_{Q} B^{(0)}}{h} \int \vec{\nabla}_{\perp} I_{l} \sin u d z=\frac{\epsilon_{C} B^{(0)}}{h^{2}} \vec{\nabla}_{\perp} I_{Q} \cos u
\end{aligned}
$$

$$
\begin{aligned}
\psi^{(1)} & =-\frac{\epsilon_{e}}{h^{2}} \vec{\nabla} I_{l} \cos u \cdot \vec{\nabla} \psi^{(0)} \\
& =-\frac{\epsilon_{e}}{h^{2}}\left\{h I_{l}^{\prime} \cos u \partial_{r} \psi^{(0)}-\frac{I_{l} l \sin u}{r^{2}} \partial_{\theta} \psi^{(0)}\right\} \\
& =-\frac{\epsilon_{l}}{h}\left\{I_{l}^{\prime} \cos u \partial_{r} \psi^{(0)}-\frac{l}{h r^{2}} I_{l} \sin u \partial_{\theta} \psi^{(0)}\right\} \\
& =\cos u\left\{-\frac{\epsilon_{e}}{h} I_{l}^{\prime} F_{\theta}\right\}+\sin u\left\{\frac{l}{h r} I_{l} F_{r}\right\}
\end{aligned}
$$

This has the esfect of producing a lubsing with helies summetry. She contaurs of $\psi^{(0)}$ brome trianguter in shape for an $l=3$ suptem. An expet colulation for $\Gamma^{\prime \prime \prime}(r, \theta)$, will nuvire the erabuation of $\vec{B} \cdot \vec{F} \psi=0$ in thich arder. 
15

III) Contivetion of $\left\langle B^{(\sigma\rangle^{2}}\right\rangle$ to $\Omega$.

$$
\begin{aligned}
& \vec{B}(\delta)=\vec{\nabla} \frac{\epsilon_{1} B^{(0)}}{h} I_{l} \sin u \\
& \vec{B}_{B}^{(j)}=\frac{\epsilon_{2} B^{(0)}}{h}\left(\hat{r} \partial_{r} I_{2} \sin u+\hat{\theta} \frac{\hat{l}_{I}}{r} \cos u+\hat{z}(-h) I_{2} \cos n\right) \\
& \hat{B}^{(\delta)}=\operatorname{Ee} B^{(0)}\left(I_{0}^{\prime} \sin n \hat{r}+\frac{l I_{c}}{h r} \cos n \hat{\theta}-I_{Q} \cos n \hat{z}\right) \\
& B^{(5)^{2}}=\epsilon_{0}^{2} B^{(0)}\left\{I_{0}^{\prime 2} \sin ^{2} u+I_{2}^{2} \cos ^{2} u\left(\frac{C^{2}}{h^{2} x^{2}}+1\right)\right\} \text {. } \\
& \left\langle B^{(\delta)^{2}}\right\rangle=\frac{1}{2} \epsilon_{i}^{2} B^{10)^{2}}\left(I_{i}^{2}+\left(\frac{h^{2}}{h^{2} r^{2}}+1\right) I_{i}^{2}\right)\langle\rangle \equiv\langle\rangle_{\frac{2 \pi}{h}} \\
& \therefore \quad \Omega=\left\langle\frac{B^{(8)^{2}}}{B^{(0)^{2}}}\right\rangle-2 k r \cos \theta \text {. }
\end{aligned}
$$




\section{References}

1. J. M. Greene and J. L. Johnson, Phys. Fiuids $\underline{4}, 875$ (1961).

2. J. L. Johnson, H. E. Dalhed, J. M. Greene, R. C. Grimm, Y. Y. Hse ih, S. C. Jardin, J. Manickam, M. Okabayashi, R. G. Storer, A. M. M. Todd, D. E. Voss, and K. E. Wejmer, J. Comp. Phys. 32, 212 (1979).

3. AEC Report - NYO 998, M..D. Kruskal

4. M. D. Kruskal and R. L. Kulsrud, Phys. Fluids 1, 265 (1958).

5. 0. Buneman, Stanford University Institute for Plasma Physics Research Report SUIPR-294 (1969).

6. J. M. Greene and J. L. Johnson in Advances in Theoretical Physics, K. A. Brueckner, ed. (Academic Press, N. Y. 1965) Vol. 1, P. 195.

7. J. P. Friedberg, Bul1. Am. Phys. Soc. II, 24, 1051 (1979).

8. T.K. Chu, H.P. Furth and C. Ludescher, Bult. Am. Phys. Soc. II, 24, 956 (1979).

9. J. L. Johnson, Max Planck Institut für Plasmaphysik Report IPP $6 / 162$ (1977).

10. D. R: Dobrott, D: D. Nelson; J. M. Greene, A. H. Glasser, M. S. Chance and E. A. Frieman, Phys. Rev. Lett. 39, 943 (1977).

11. R. C. Grimn, J. M. Greene, J. L. Johnson in Methods of Computational Physics, J. Killeen, ed. (Academic Press, N. Y. 1976) Vol. 16, P. 253.

12. D. R. Dobrott, J. M. Greene and J. L. Johnson, Phys. Fluids 13, 795 (1970). 
Table 1.

Basic Equations

\section{Equations}

$\rightarrow+\rightarrow$

$J \times B=\nabla P$

$\vec{\nabla} \vec{B}=4 \pi$

$\nabla \times B=4 \pi \quad \mathrm{J}$

$\rightarrow \vec{\nabla}$

$\nabla: B=0$

$\vec{B} \cdot \vec{\nabla}=0$.

$\overrightarrow{B^{0}}=B^{0} \vec{\zeta}$

$\overrightarrow{B^{\delta}}=\vec{\nabla} \sum \frac{B^{0} \varepsilon_{2}}{h} I_{\ell}(h r)$

$$
\cdot \sin \left(2 \theta-h_{5}+\phi_{2}\right)
$$

$\vec{B}^{x}=B^{0} \times r \cos \theta \dot{\vec{r}}$
Coments

Equilibria Condition

Maxwell Equations
Establishes as the coordinate of the magnetic surfaces.

Zero order magnetic field

First order field derived from Laplace's equation in a helical geometry. The system is periodic over a length $2 \pi / h$. $\varepsilon_{p}$ is the mode amplitude (replace $h$ by ph and sum over $p$ for external windings with more than one wavelength).

Second order field due to curvature. 


$$
\overrightarrow{s^{3}}=\frac{-4 \pi D^{8}}{B^{0}}\left(\varphi^{0}\right) \dot{\zeta}
$$$$
\text { Detine: } \sigma=\vec{J} \cdot \vec{B} / \vec{B} \cdot \vec{B}
$$$$
\vec{j}=\vec{j}_{1}+\overrightarrow{0}
$$$$
\overrightarrow{8} \cdot \overrightarrow{\nabla \sigma}=\left(\nabla \overrightarrow{B^{2}} \times \vec{B}\right) \cdot \overrightarrow{\nabla p / B^{4}}
$$$$
\left(\vec{B} \cdot \overrightarrow{v_{0}}=2(\vec{B} \times \overrightarrow{\nabla p}) \cdot \vec{x} / \mathrm{s}^{2}\right)
$$$$
\overrightarrow{B^{\sigma}}=\vec{\nabla} \times\left(A^{\sigma}, \vec{\zeta}\right)
$$$$
\nabla^{2} A^{\sigma}=-4 \pi B^{0} \sigma^{\lambda \lambda}
$$

$y^{0}=\frac{\pi B^{0}}{k} \sum_{2, \pi}^{\sum} \frac{\varepsilon_{l} \xi_{m}}{h^{2} r} m I^{\prime} L(h r) I_{m}^{\prime}(h r)$

$\cdot \cos \left[\dot{[}(2-m) \theta+\phi_{L}-\phi_{\pi}\right]-\frac{2 \pi}{x} A^{\sigma}$

$$
\begin{gathered}
\sigma^{\lambda \lambda}=\frac{-2 \pi}{K B^{0}}: \rho^{B^{1}}\left(\psi^{0}\right) \Omega(r, \theta) \\
+G\left(\varphi^{0}\right) .
\end{gathered}
$$

$$
\Omega=1 / 2 \varepsilon_{2, m}^{E} \varepsilon_{q} \varepsilon_{m} I ! 2(h r) I \operatorname{m}(h r)
$$$$
\left[1+\left(2 m+h^{2} r^{2}\right) I^{2}(h r) I^{m}(h r)\right]
$$$$
\text { - } \cos [(l-m) \theta+\phi q-\phi m]-2 x r \cos
$$

$p=p_{\text {ixax }}^{3}\left(1-q^{0}\right)$

$G=G_{0}+\frac{x G 7}{8 \pi^{2} B^{0}} \varphi^{0}$

$$
G\left(r_{1}, r_{2}\right)=\frac{1}{2 \pi} \ln \left(r_{2} / r_{1}\right)
$$

Second order field - pressure balance. Scalar parallel current

via $\nabla \cdot \vec{j}=0$

$\vec{k}$ represents curvature. This is a vanishing net drift due to charge. separation.

In lowest order $\sigma$ is independent of $\zeta$ via the equation above. We denote our vector potential by $A \sigma$ and note that it is of second order.

Two dimensional equation : Compare with Grad - Shafranov Equation.

The magnetic surfaces are labelled by y and it may be identified with the poloidal flux function in zeroth order. The condition of periodicity is used to establish this result.

$\Omega$ is a geometrical expression and $G$ is an arbitrary function which ought to be chosen to be consistent with a reasonable current profile. $\Omega$ represents the locai curvature averaged over the short wavelength.

$$
I^{2}(x) \equiv \frac{I L(x)}{x I^{\prime} L(x)}
$$

Assumed pressure profile, current profile. An analytical solution is, found in J. M. Gresne and J. L. Johnson. Green's function used to calculate contribution to flux at $r_{1}$ due to current at $r_{2}$. 
Table 2.

\section{Comparison with Tokamak}

$$
\begin{aligned}
& \vec{J} \times \vec{B}=\vec{\nabla} P \\
& \vec{\nabla} \times \vec{B}=\vec{J}^{\dagger} \\
& \vec{\nabla} \cdot \vec{B}=\overrightarrow{0}
\end{aligned}
$$

\section{Tokamak}

Multipolar Fields

$$
\begin{aligned}
& \Psi=2 \pi \times A \phi \Psi^{(0)}=\frac{\pi}{\kappa} \varepsilon_{m}{ }^{2} \frac{m}{h^{2} r} I_{m}^{\prime}(h r) I_{m}(h r)-\frac{2 \pi}{k} A(\sigma) \\
& \nabla \star \Psi=2 \pi \times J_{\phi} \nabla^{2} A(\sigma)=-4 \pi B(0)_{\sigma}(\lambda \lambda) \\
& \nabla^{*} \equiv\left(x \partial_{x} \frac{1}{x} \partial_{x}+\partial_{z z}\right) \nabla^{2}=\left(\partial_{x x}+\partial_{z z}\right) \\
& J_{\phi}=-2 \pi\left(\frac{d}{d \Psi} p(\Psi) x+\frac{R^{2}}{2 x} \frac{d}{d \Psi}\left(g^{2}(\Psi)\right) \sigma=-\frac{2 \pi}{k} \frac{d p(\Psi(0))}{d \Psi(0)} \Omega(r, \theta)+G(\Psi(0))\right. \\
&\left.\Omega=-\frac{2 r}{R} \cos \theta+<B(\delta)^{2}\right\rangle
\end{aligned}
$$

$+\vec{\nabla} \times \vec{B}=4 \pi \vec{J}$ is used for multipolar fields. $B^{(0)}=1$ in the above. 
Table 3

Comparison of Analytical \& Computational Solutions

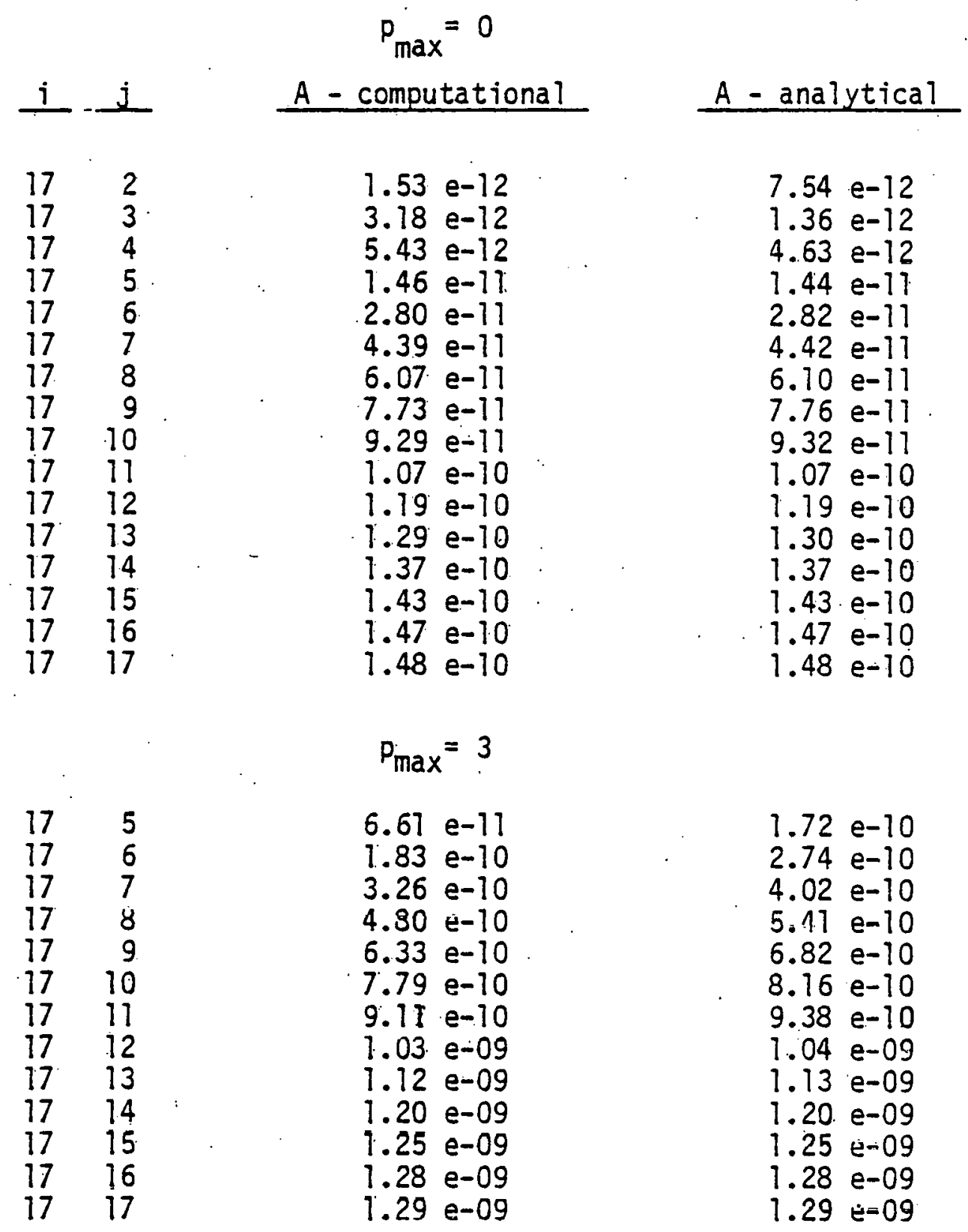


Legends for Figures

Fig. 1. Coördinates of the torus of major radius $R$, minor radius a. Fig. 2. Coordinates for the grid.

In Fig. $4 a-4 h$ the pressure and current profiles used are:

$L \equiv$ Limiter

$$
\begin{aligned}
& p=p_{0}\left(\frac{\psi_{L}-\psi}{\psi_{L}-\psi_{m}}\right) \\
& G=G_{0}+\frac{k G 1}{8 \pi^{2} B^{(0)}}\left(\frac{\psi_{L}-\psi}{\psi_{L}-\psi_{m}}\right)
\end{aligned}
$$

$M \equiv$ Magnetic Axis

In all other figures the profiles used are:

$$
\begin{aligned}
& p=p_{0}\left(1-\alpha \psi^{(0)}\right) \\
& G=G 0+\frac{k \cdot G}{8 \pi^{2} B^{(0)}} \psi^{(0)}
\end{aligned}
$$

Fig. 3a. Comparison of Analytical and Computational Solutions for $\beta=0$.

$A$ vs. $r$. $j$ values are given on the line $j=17$.

Fig. 3b. Comparison of Analytical and Computational Solutions for $\beta \cong 1 \%(p 0=3)$. A vs. $r$. $j$ values are given on the line $i=17$.

Fig. 4a. Contours of poloidal magnetic flux: $\beta=0$.

Fig. 4b. Contours of plasma current along field lines: $\beta=0$.

Fig. 4c. Current profile at the grid midplane: $\beta=0$.

Fig. 4d. Linear pressure profile.

Fig. 4e. Contours of poloidal magnetic flux: $\beta=2 \%$. 
22

Fig. Af. Contours of plasma current along field lines: $\beta=2 \%$.

Fig. 4g. Current profile at the grid midplane: $\beta=2 \%$.

Fig. 4h. Pressure profile at the grid midplane: $\beta=2 \%$.

Fig. 5. ' Position of magnetic axis vs. Pax

$$
p=p_{\max }(1-\alpha \Psi(0))
$$

Vertical axis measures distance in meters from toroidal axis.

Fig. 6. Position of Magnetic Axis vs. Current in Vertical Field Coils.

An approximately. vertical field is estabilshed with two coils, placed at $x=22, z=5$ meters. The magnitude of the field is proportional to the current, given approximately by $B \cong 2 I / r$. 


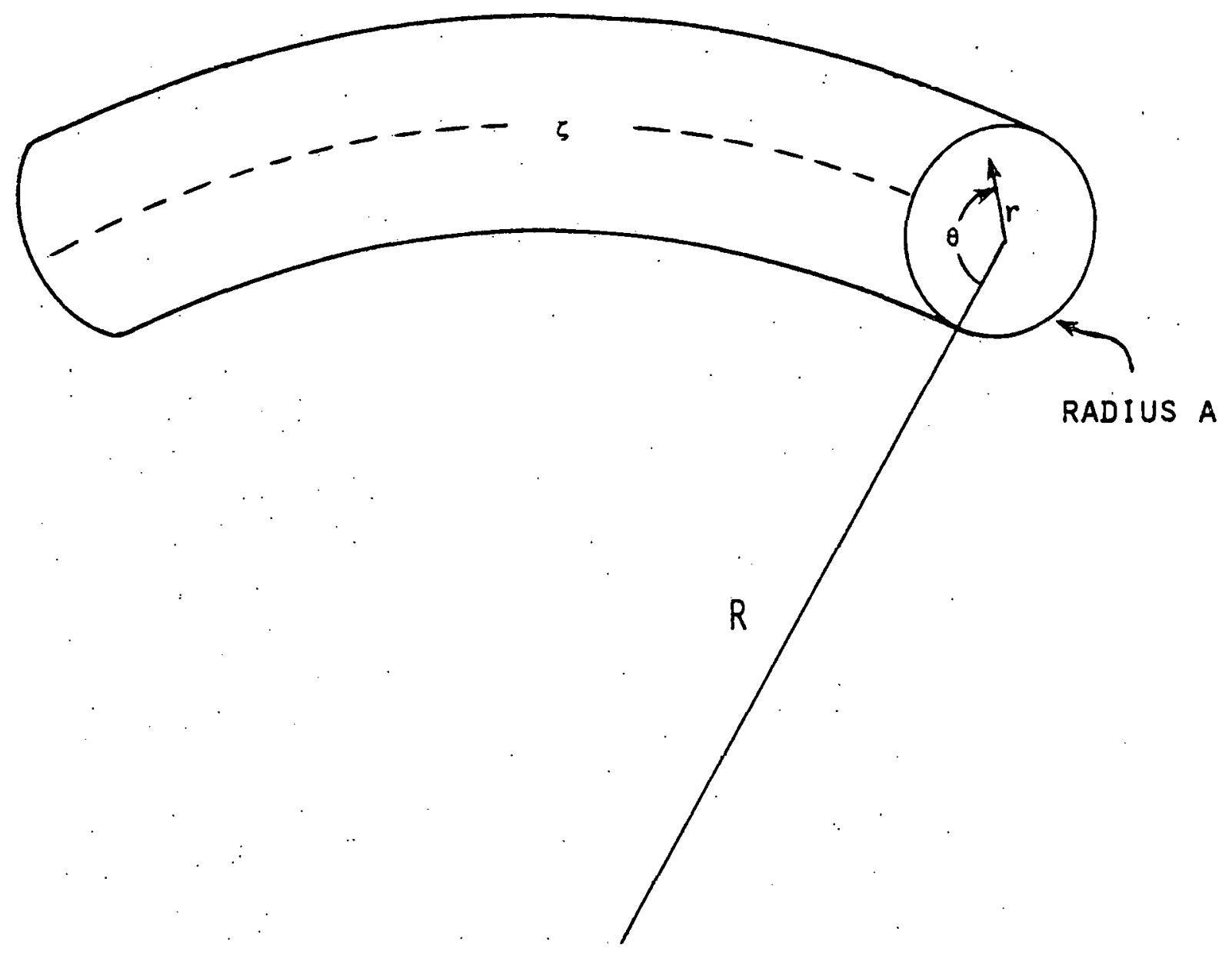

Fig. 1 .

COORDINATES ON THE TORUS 
vertical field coil

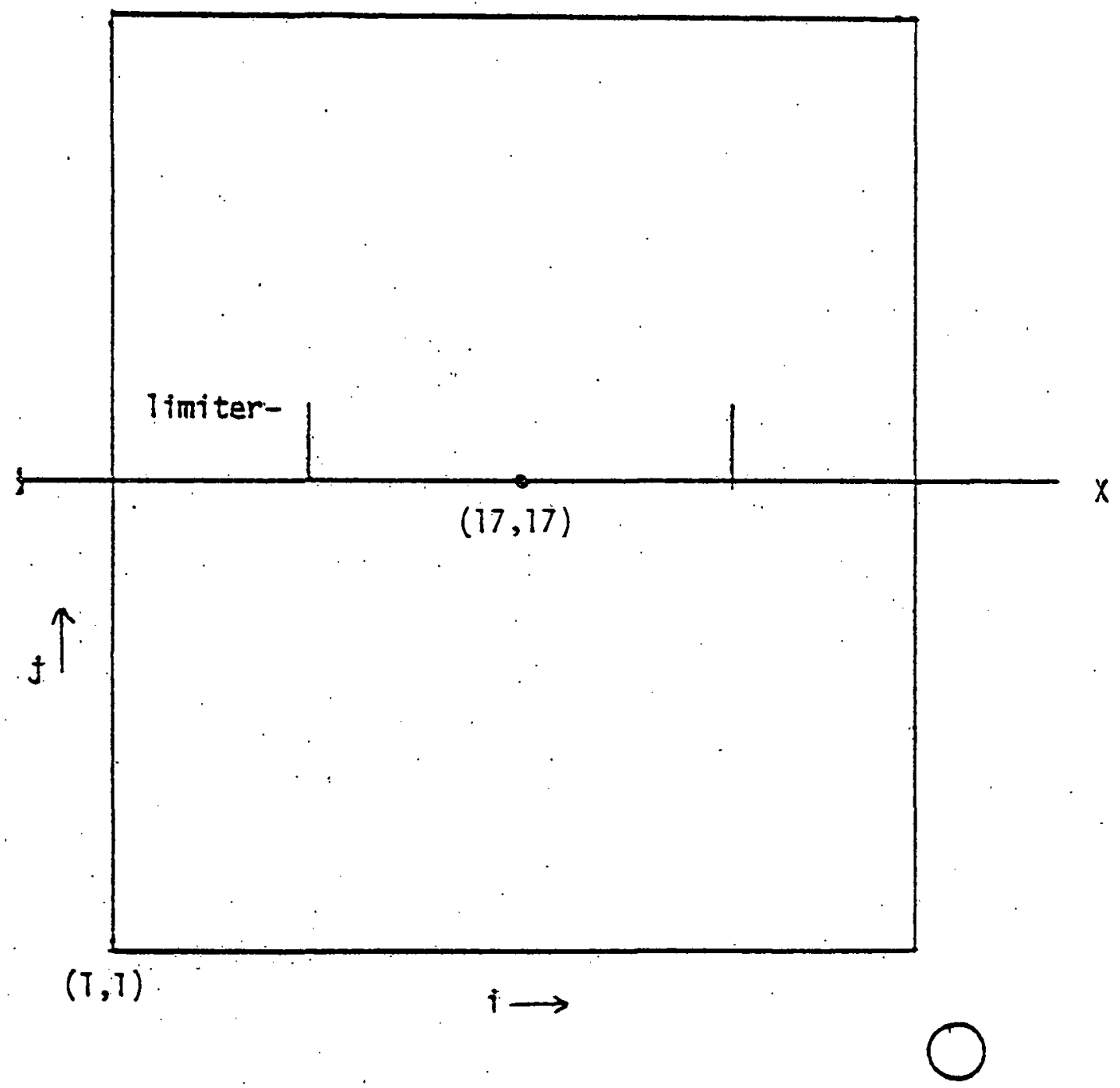

Fig. 2 . 
Fig. 3a.

COMPARISON OF ANALYTICAL AND COMPUTATIONAL SOLUTIONS FOR $\beta=0$

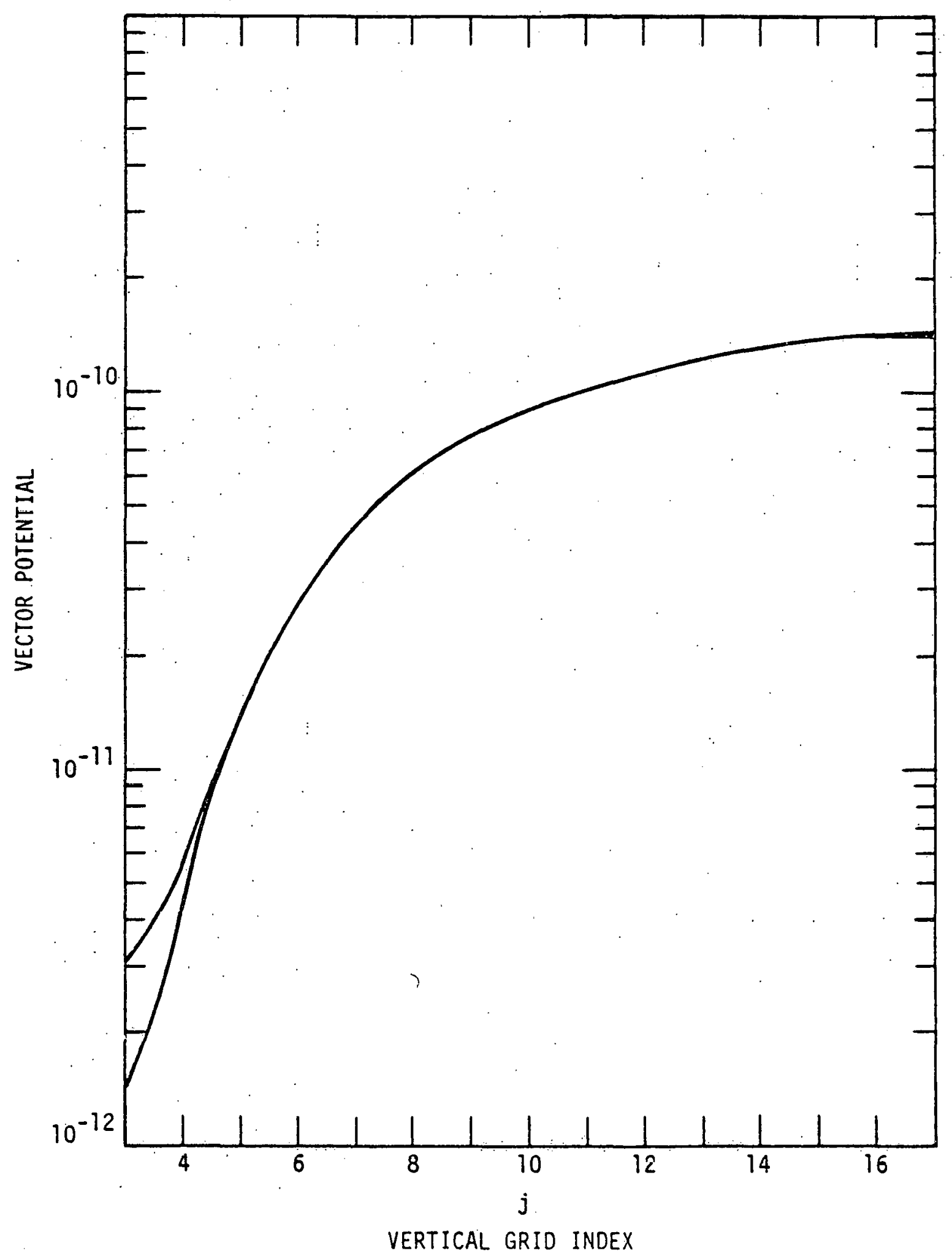


Fig. 3b.

COMPARISON OF ANALYTICAL AND COMPUTATIONAL SOLUTION FOR $\beta \cong 1 \%$

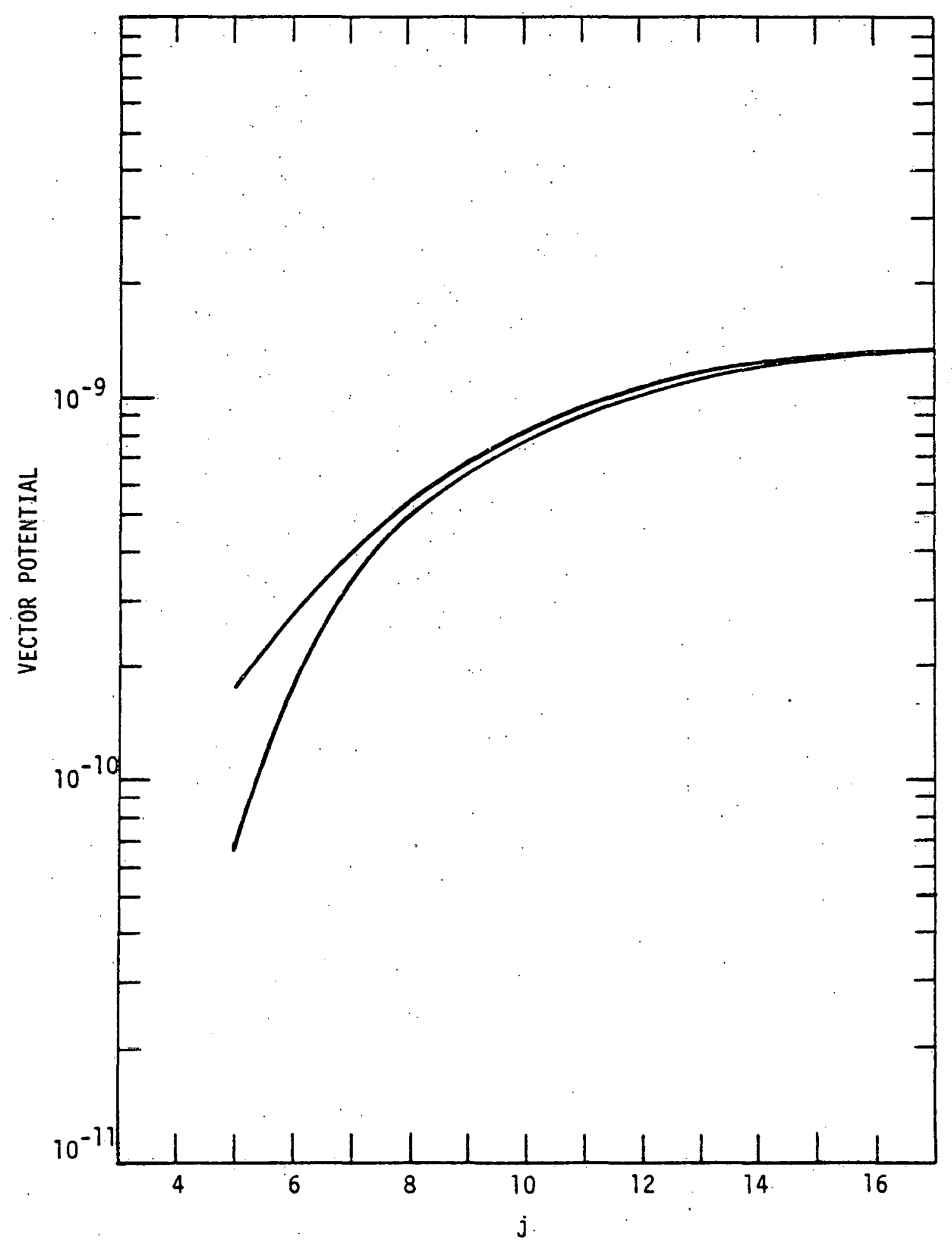




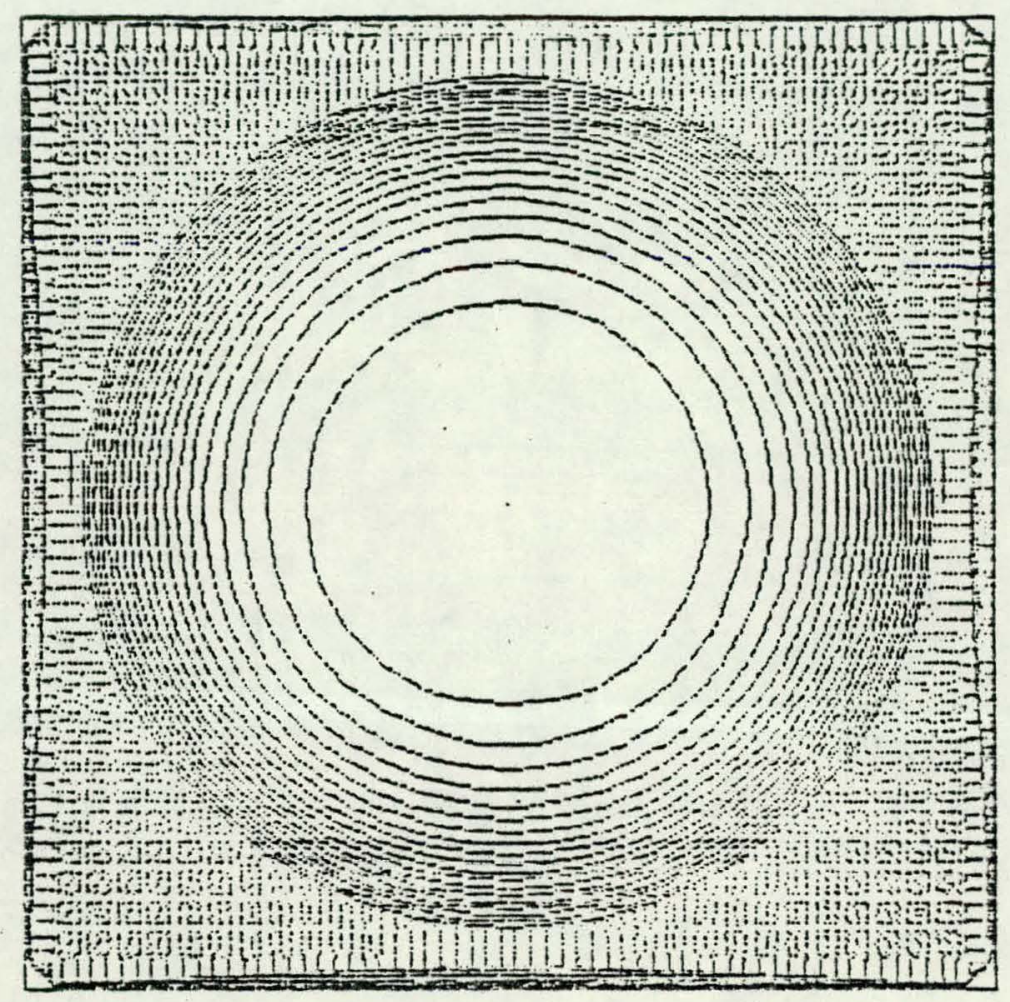

$x$

Fig. $4 a$. 


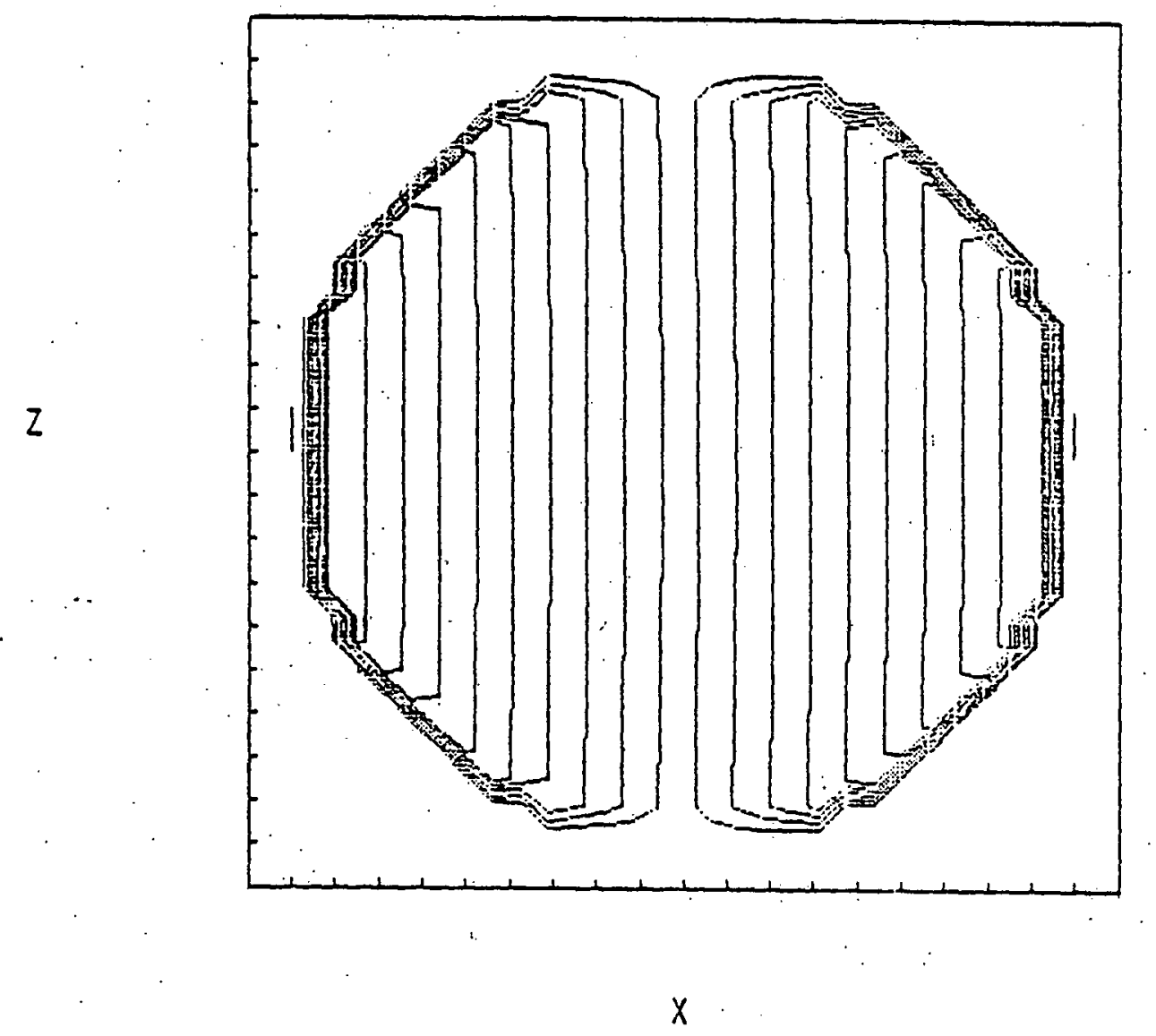

Fig. 4b. 


\section{Current}

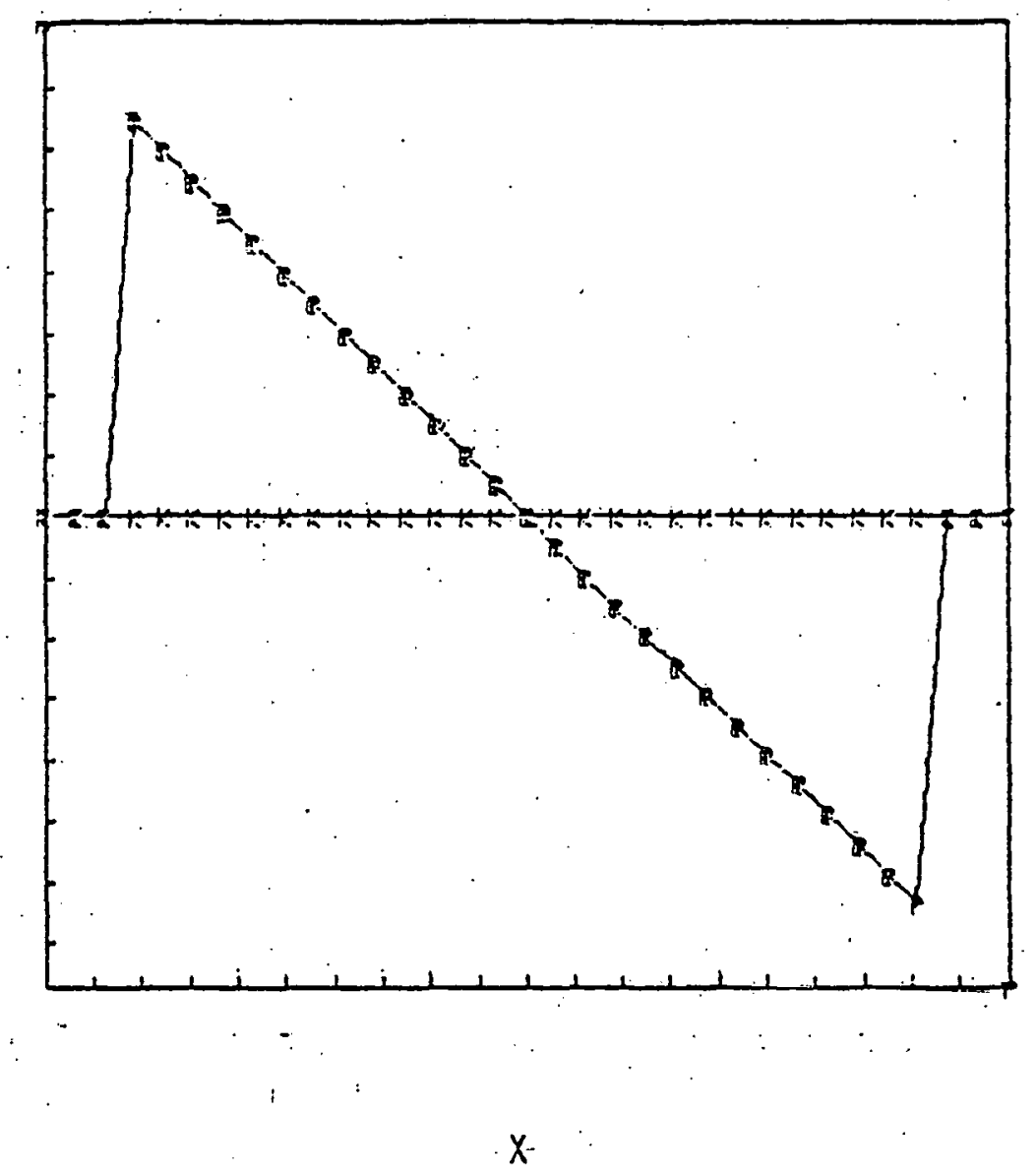

Fig. $4 c$. 
Pressure

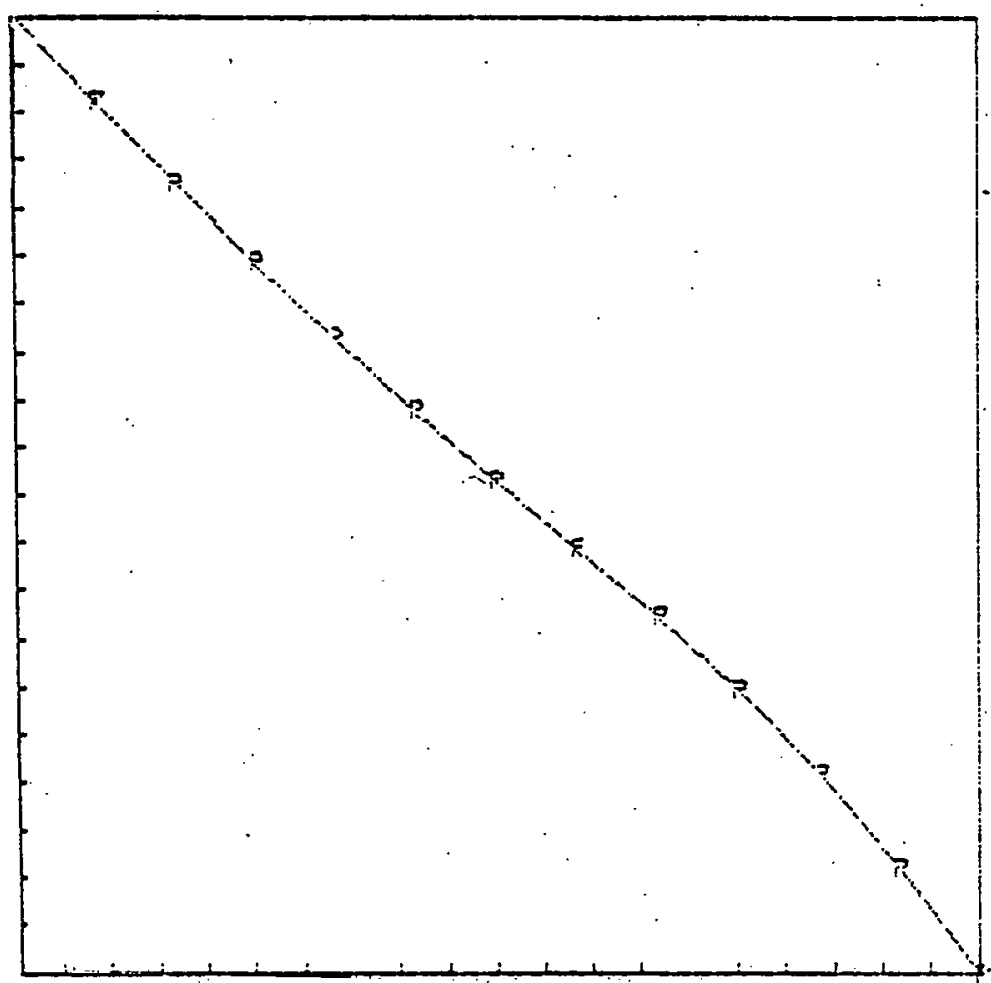

PSI

Fig. 4d. 


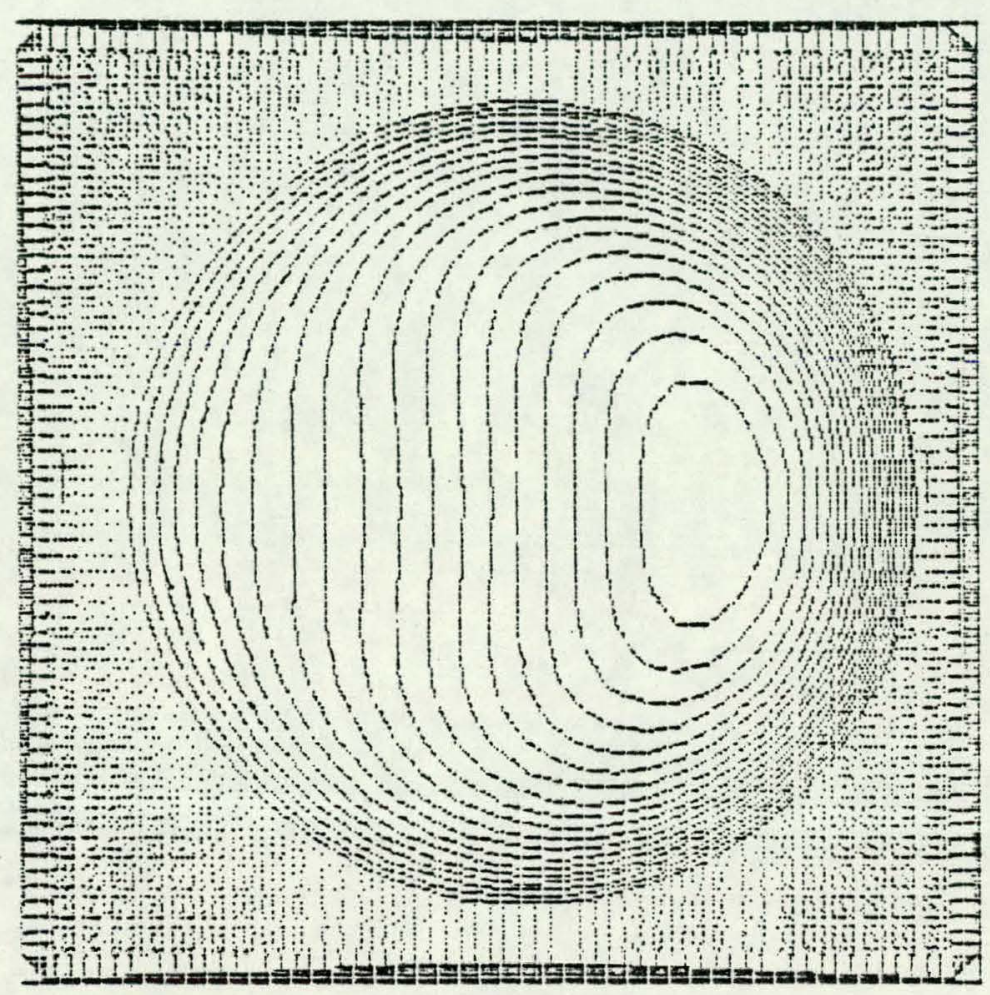

$x$

Fig. 4e. 


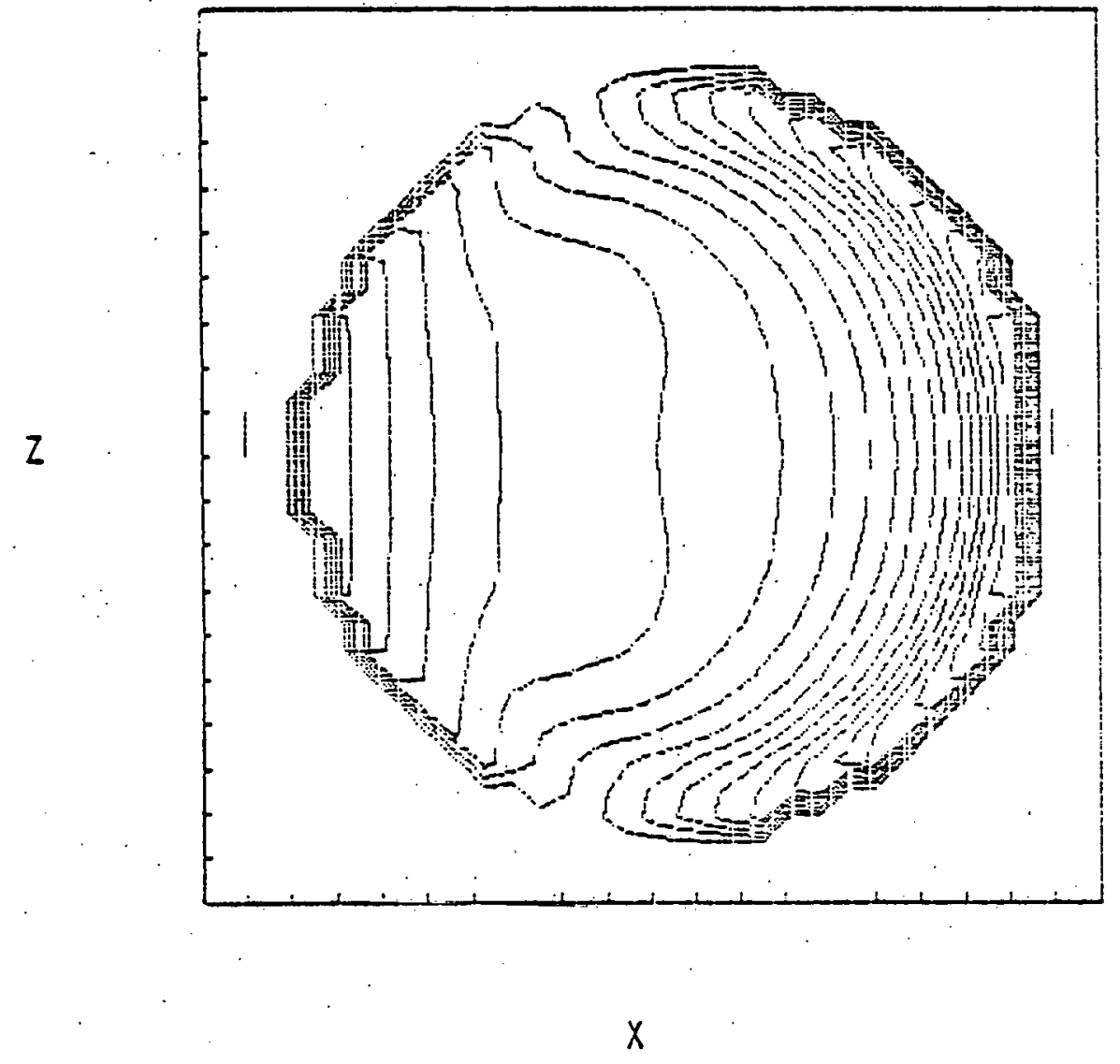

Fig. $4 f$. 


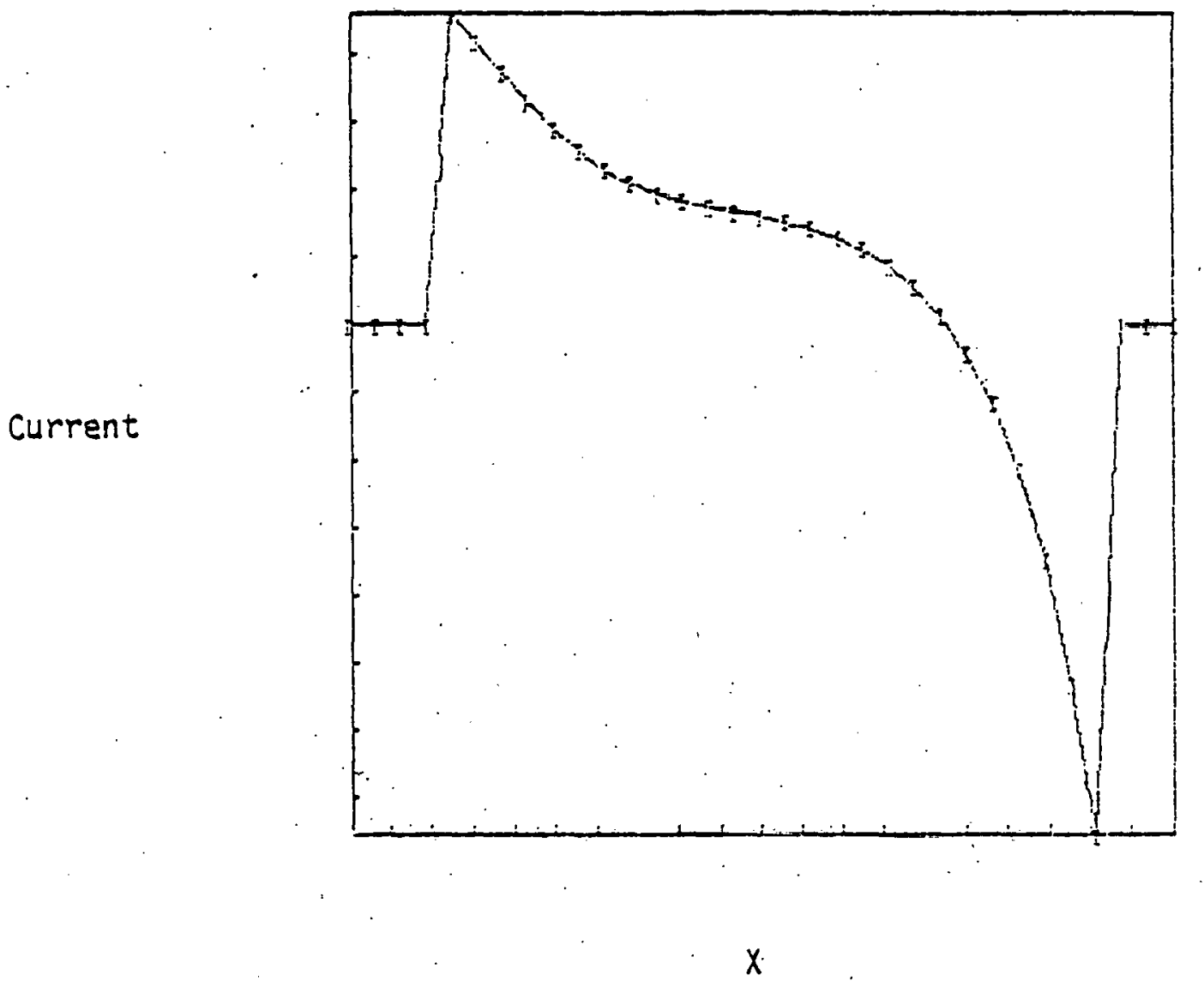

Fig. 4 . 


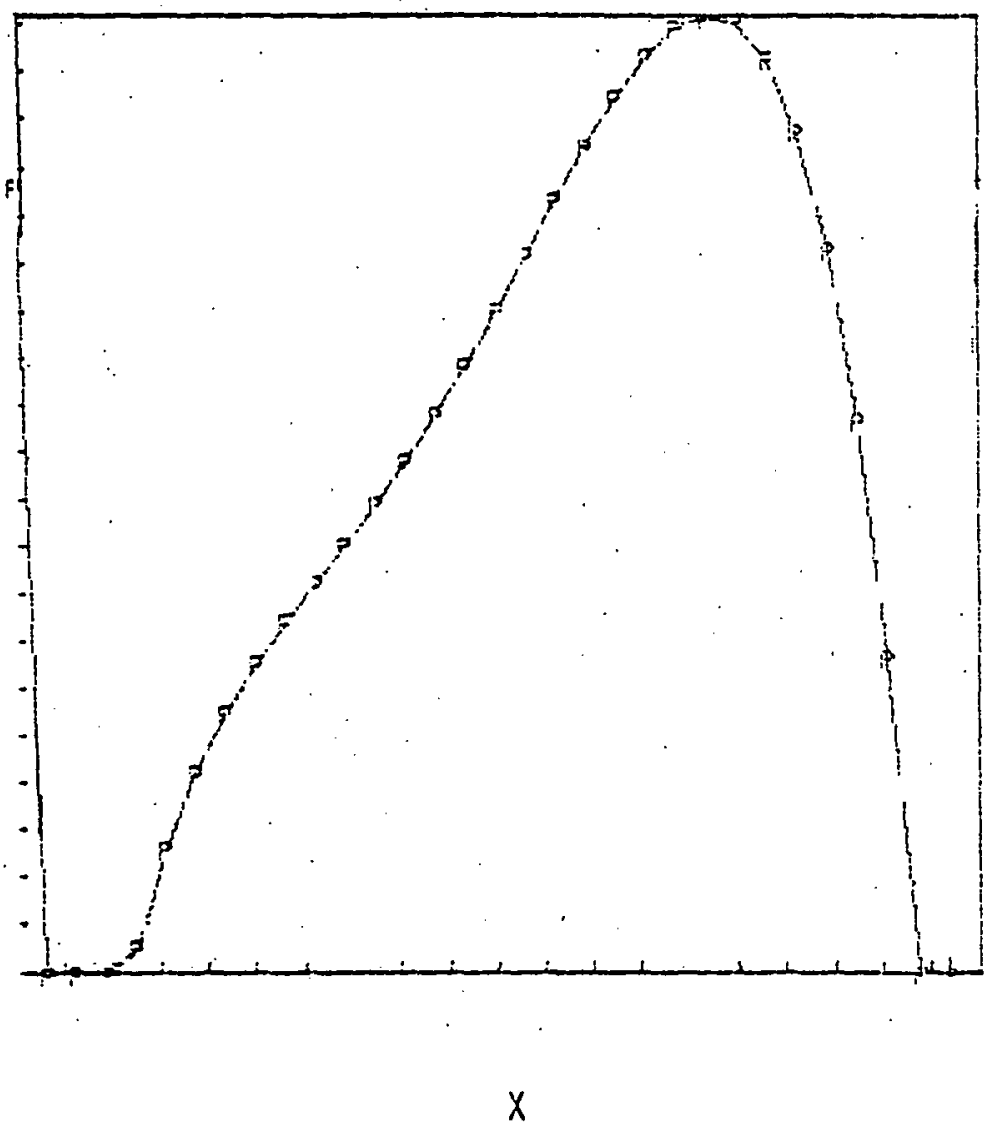

Fig. 4 h. 


$$
17
$$






